\title{
Progenitor Evolution and Dark Energy Time Variation from CLASH SNe Ia
}

\author{
Enikő Regös and CLASH Collaboration
}

CERN, ELTE

\begin{abstract}
The nature and timescales behind the growth of the white dwarf toward the Chandrasekhar mass are not known. The two leading competing scenarios for Type Ia supernovae (SNe Ia) are accretion from a companion [single degenerate (SD)] or merger with another white dwarf [double degenerate (DD)]. Measurement of the SNe Ia delay time distribution could distinguish between these scenarios. Possibly both channels operate, on short (SD) and long (DD) time scales. A supernova search in parallel with our Cluster Lensing And Supernova survey with Hubble extends the Hubble diagram of SNe Ia to $z>1.5$, probing progenitor evolution and testing the constancy of dark energy (DE) with time. We use HST ACS to detect SNe Ia at $1<z<1.5$ and WFC3 to find SNe Ia at $1.5<z<2.5$, thus providing constraints for the variation in the DE equation of state. This redshift epoch provides the unique chance to test SNe Ia distance measurements for the deleterious effects of evolution independent of our ignorance of dark energy. Our program provides the first measurement of the SNe Ia rate at $z \sim 2$.
\end{abstract}

Keywords. supernovae: general - cosmology: cosmological parameters, distance scale

\section{Overview}

The natures of the fundamental constituents of the universe are not well understood. The composition of the universe has proven to be far more intriguing than we thought it to be, even only 15 years ago. It is a "dark" universe where $23 \%$ of the mass-energy is made up of as yet undetected, weakly interacting, non-baryonic particles (dark matter), and $73 \%$ is associated with as yet unknown physics that drives accelerated cosmic expansion (dark energy). The Multi-Cycle Treasury Program presents an opportunity to make major progress on understanding the physics behind these phenomena. We were awarded 524 orbits of HST time to the Cluster Lensing and Supernova Survey with Hubble $(C L A S H)$. Our survey uses panchromatic imaging from the new Wide Field Camera 3 (WFC3) and the restored Advanced Camera for Surveys (ACS).

In parallel we use ACS and WFC3 infrared (IR) channel to detect Type Ia supernovae in the range $1<z<2.5$, which is uniquely accessible from space. The SNe Ia are used to improve the limits on the redshift variation of the dark energy equation of state. We investigate if SNe Ia show evidence for significant evolution at high redshift. To follow up on the SNe Ia we discovered, we have a joint reserve program with the Cosmic Assembly Near-IR Deep Extragalactic Legacy Survey (CANDELS). We present the fields and images of selected supernovae of various types in Figures 1 and 2, and spectroscopy of some of their host galaxies in Figure 3 .

Our SN search extends the Hubble diagram to $z>1.5$, testing the constancy of dark energy with time and probing progenitor evolution. Considering kinematics in curved space, Type Ia supernovae are used as galaxy distance indicators to derive cosmological parameters. 


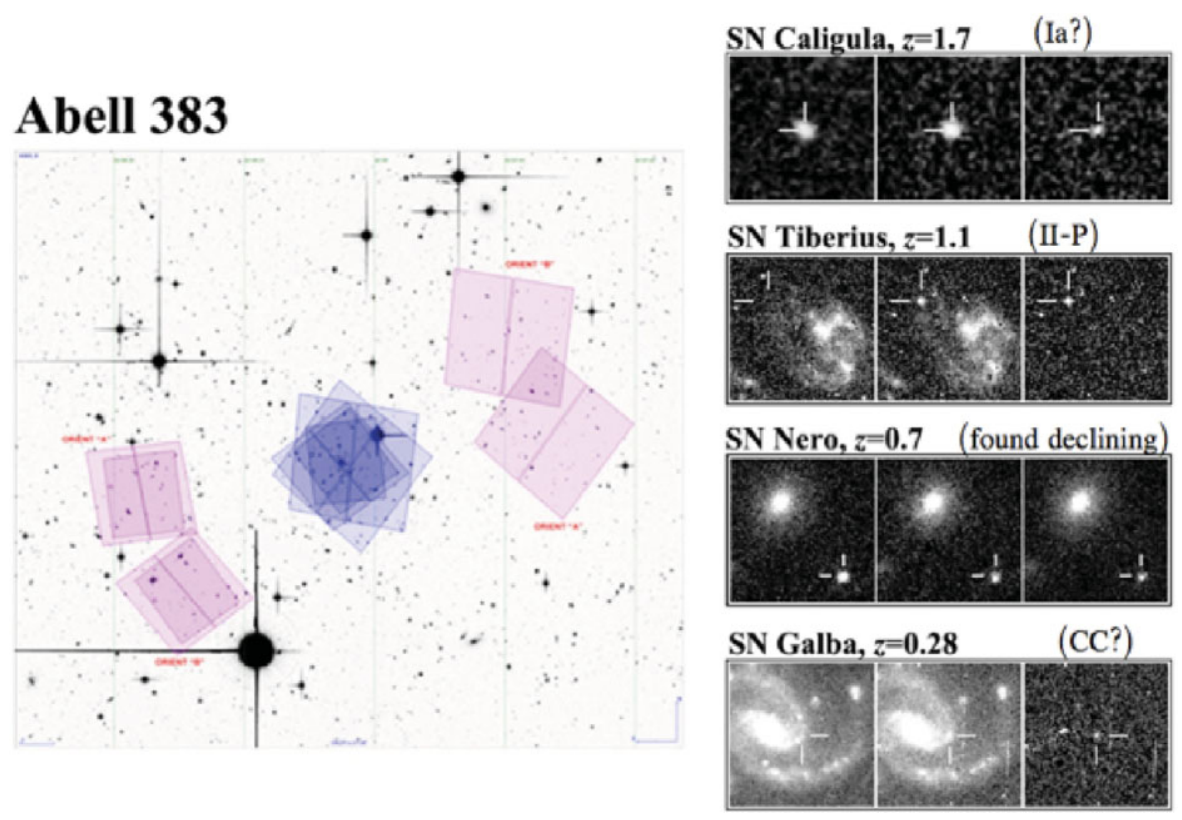

Figure 1. SNe Caligula, Tiberius, Nero and Galba in Abell 383.
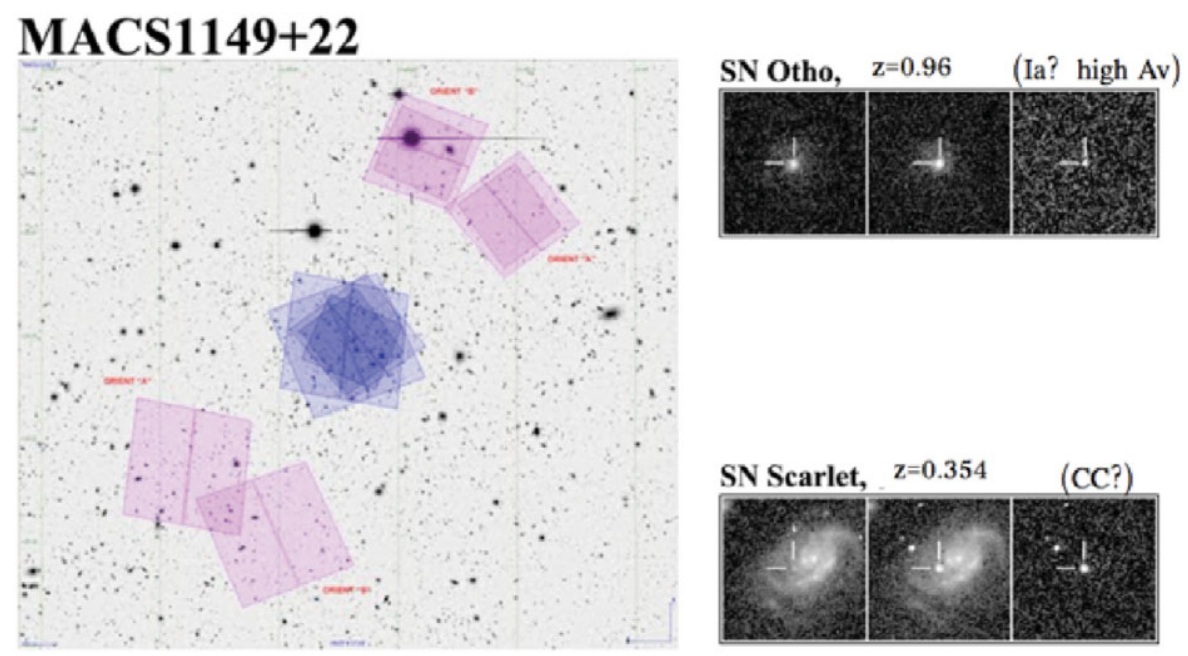

Figure 2. SNe Otho and Scarlet in MACS $1149+22$.

In the SD scenario, the secondary star evolves and transfers mass to its WD companion. This secondary star can be a slightly evolved main-sequence star, or a giant in the case of a symbiotic progenitor. Previously the SD scenario was disfavored because it was believed nova explosions prevented the WD to gain mass, but now there are new supersoft X-ray source observations which favor the SD scenario (see Kato, these proceedings). Edge-lit detonations and merging CO WDs contribute to the DD channel.

In population studies of SD and DD scenarios of SNe Ia, there is a relationship between the rate and delay time (see Nelemans, these proceedings). It is believed that SD progenitors dominate at prompt delay times while DD progenitors primarily contribute at late delay times. Our results are consistent with a long delay corresponding to the DD 


\section{Otho+Scarlet: \\ Ground-based spectroscopy confirms the photo-z estimates}
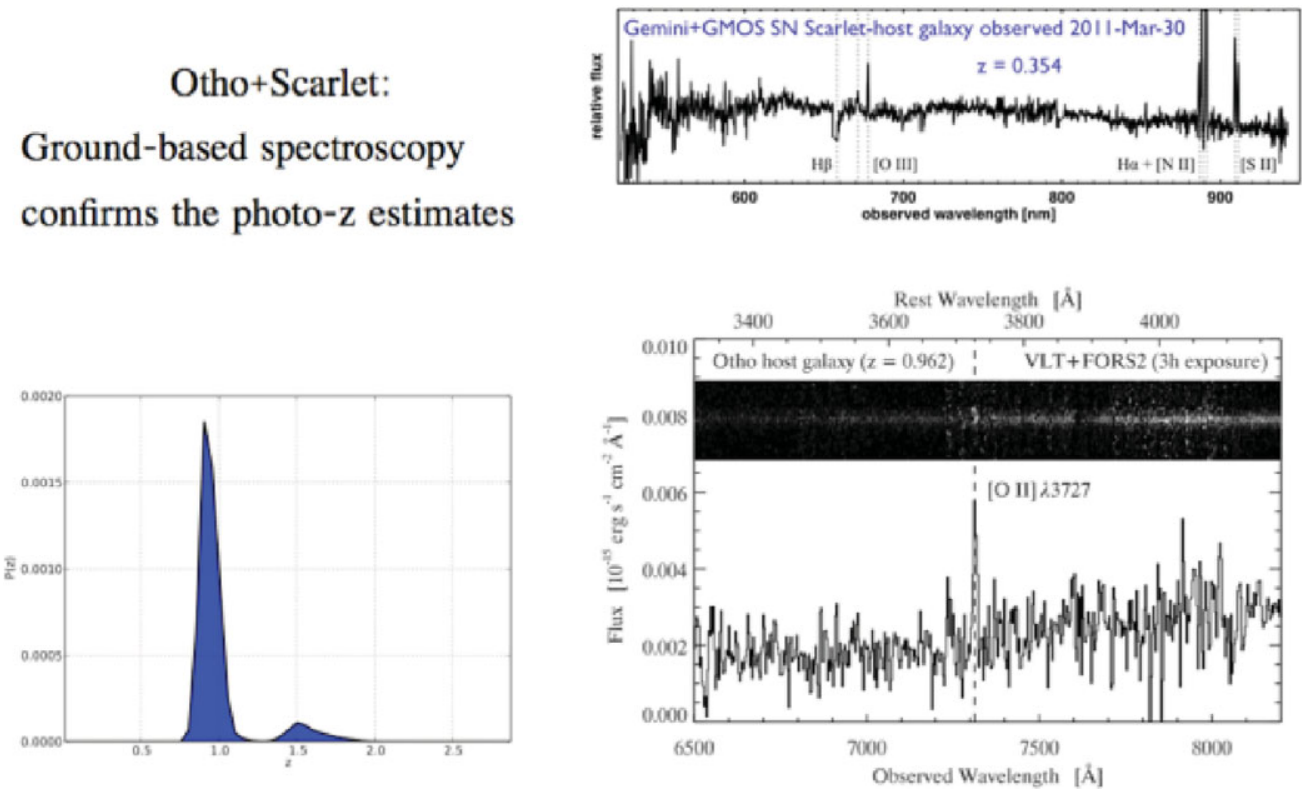

Figure 3. Spectroscopy of SNe Otho and Scarlet.

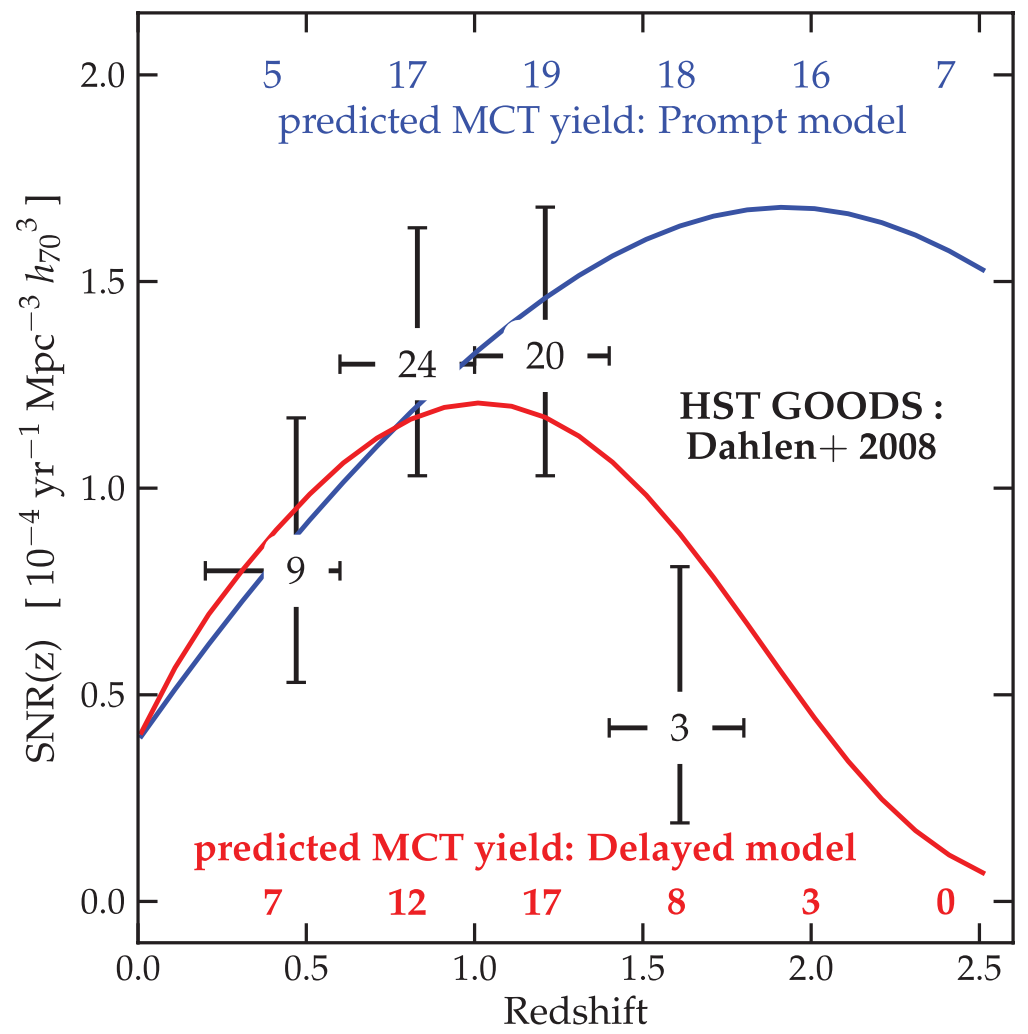

Figure 4. Projected HST SNe Ia rates discrimination.

scenario (red curve in Figure 4). The blue curve in Figure 4 corresponds to a small delay and the SD scenario. 